\title{
RNA-sequencing and Mathematical Modeling Identify Suite of Light-sensitive Circadian Genes in an Orb-web Weaving Spider
}

\section{Natalia Toporikova}

Washington and Lee University

\section{Wenduo Cheng}

Duke Kunshan University

\section{Leyuan Qian}

Duke Kunshan University

\section{Andrew Mah}

Washington and Lee University

Thomas Clarke

Washington and Lee University

Thomas C. Jones

East Tennessee State University

\section{Darrell Moore}

East Tennessee State University

Nadia A. Ayoub ( $\sim$ ayoubn@wlu.edu )

Washington and Lee University

\section{Research Article}

Keywords: Circadian rhythms, Metazygia wittfeldae, Araneidae, entrainment, RNA sequencing, de novo transcriptomics, mathematical modeling

Posted Date: November 11th, 2021

DOI: https://doi.org/10.21203/rs.3.rs-1036447/v1

License: (c) (i) This work is licensed under a Creative Commons Attribution 4.0 International License.

Read Full License 
1 RNA-sequencing and mathematical modeling identify suite of light-

2 sensitive circadian genes in an orb-web weaving spider

3

4 Natalia Toporikova ${ }^{1, *}$, Wenduo Cheng ${ }^{2}$, Leyuan Qian $^{2}$, Andrew Mah ${ }^{1,3}$, Thomas Clarke ${ }^{1}$, Thomas C.

5 Jones $^{4}$, Darrell Moore ${ }^{4}$, Nadia A. Ayoub ${ }^{1 *}$

6

$7 \quad{ }^{1}$ Department of Biology, Washington and Lee University, Lexington, VA, 24450, USA

$8{ }^{2}$ Division of Natural and Applied Sciences, Duke Kunshan University, Kunshan, Jiangsu, 215300,

9 People's Republic of China

$10 \quad{ }^{3}$ Center for Neural Science, New York University, New York, New York, 10003, USA

$11{ }^{4}$ Department of Biological Sciences, East Tennessee State University, Box 70703, Johnson City, TN,

1237604, USA

13

$14 *$ *uthors for correspondence:

15 Natalia Toporikova toporikovan@wlu.edu

16 Nadia A. Ayoub ayoubn@ wlu.edu 


\section{Abstract}

\section{Background}

19 Most organisms rely on a molecular circadian clock to orchestrate a wide range of physiological

20 processes to match the 24-hour day. These molecular clocks are typically based on a negative

21 feedback loop among a small set of proteins that govern the circadian output. Light or other

22 environmental conditions can reset the circadian clock, but true circadian behaviors continue to

23 cycle even in constant darkness, with an intrinsic period called the free-running period (FRP).

24 Spiders have unusual FRPs, with some species having extremely short FRP (e.g. 18 hours for

25 trashline orb weaver), and many having highly variable FRPs (intraspecific variation of up to 10

26 hours). In the absence of any genetic model of circadian rhythms in spiders, we developed a

27 mathematical model to optimize experimental conditions for identifying circadian genes that also

28 respond to light cues.

\section{Results}

30 Our mathematical model involved a single gene that encodes a protein that inhibits its own

31 transcription. In our model, light degrades the circadian transcript, which allows a broad range of

32 FRPs to be entrained to a 24-hour day. Our model predicted that exposing spiders to a pulse of

33 light in the middle of the night would cause a pattern of expression between two later time points

34 that was opposite the pattern exhibited by spiders who did not receive a pulse of light. RNA-

35 sequencing of four groups of adult female orb weaving spiders, Metazygia wittfeldae, under

36 these experimental conditions resulted in 528 significantly differentially expressed (DE)

37 transcripts between the two collection times or between the light pulse and no light pulse. 
38 Consistent with our model, we found a cluster of transcripts with the flipped pattern of

39 expression between the two collection times, dependent on the application of light.

\section{Conclusions}

41 Our DE transcripts represent the first genetic evidence for circadian output in spiders.

42 Furthermore, those transcripts with a flipped pattern of expression represent prime candidates for

43 light-sensitive circadian genes, which may be involved in entraining the circadian clock to light.

44 Functions of these genes varied from growth and development to reproduction to gene

45 regulation, consistent with other circadian systems.

\section{Keywords}

48 Circadian rhythms, Metazygia wittfeldae, Araneidae, entrainment, RNA sequencing, de novo 49 transcriptomics, mathematical modeling

\section{$51 \quad$ Background}

52 Most organisms rely on a molecular circadian clock to orchestrate a wide range of

53 physiological processes [1]. Interactions among several key proteins form multiple positive and

54 negative feedback loops resulting in a 24-hour rhythm in expression of circadian transcripts [2].

55 Even under constant darkness (DD), oscillations in circadian gene expression generate daily

56 periodic behaviors such as locomotion, hormone release, sleep-wake cycles, and many others.

57 Such regular oscillations under DD conditions (typically within 2 hours of 24 hours), called the

58 free running period (FRP), has been documented in a diverse range of organisms [3-5].

59 Circadian oscillations are also synchronized by external stimuli (zeitgebers), of which light is the 
60 strongest. In alternating light-dark environments (LD), the light-sensing system modifies

61 expression of one or more circadian genes, adjusting (or entraining) the FRP to a LD period [6].

62 In the well-studied circadian model, the fruit fly Drosophila melanogaster, the circadian

63 "clock" is controlled via a negative feedback loop, which contains transcriptional factors timeless

64 (tim), period (per), cycle (cyc) and Clock (clk). Proteins CLK and CYC form a complex that acts

65 as a transcriptional activator for per and tim genes. In early evening, concentrations of PER and

66 TIM in the cytoplasm increase, and these two proteins form a PER-TIM complex, which enters

67 the nucleus and depresses their own promoter regions by interacting with CYC-CLK, thereby

68 completing the negative feedback loop. When the PER-TIM heterodimer is degraded through

69 double-time (DBT) protein-dependent phosphorylation, CYC-CLK activates gene transcription

70 once again [7]. The fruit fly can receive information about light through the visual system via

71 activation of cryptochrome 1 (CRY1), a blue-light sensitive photoreceptor. At the end of the

72 night and in early morning, there is a lower concentration of TIM, and during the light phase

73 CRY1 binds with TIM, preventing PER-TIM formation and so causing a delay in accumulation

74 of that complex. As CRY1 degrades TIM, PER-TIM complex cannot be formed, thus relieving

75 promoter depression, stimulating CYC-CLK activity and tim, per gene transcription. Newly

76 synthesized TIM resets clocks in the beginning of the day or returns it to the initial state without

77 light (Kistenpfennig et al. 2012). In mammals and some of the arthropods, the PER/TIM

78 heterodimer indirectly blocks the function of CLK/CYC through an additional protein,

79 cryptochrome 2 (CRY2) $[8,9]$.

80 Despite the theoretical and experimental expectations for FRP to closely match the 24-hour

81 day, some spider species have remarkably short and variable FRPs of locomotor activity in

82 constant dark. A standout example is the trashline orb-weaving spider Cyclosa turbinata 
83 (Araneidae), which has a mean FRP of 18.7 hours in DD [10]. This is the shortest known

84 naturally occurring FRP, even shorter than ones caused by lab-induced mutations, such as the

85 hamster tau mutant (20 hours [11]) and the fruit fly $\mathrm{per}^{S}$ mutant (19 hours [12]). Other species of

86 spiders have been found to have extreme variation in FRP among individuals. For example, the

87 ranges of FRPs exhibited by three cobweb weaving spider species (Theridiidae) were 19-23.5 h

88 in the common house spider P. tepidariorum (mean FRP $=21.7 \mathrm{~h}$ ), 20-29 h in the subsocial

89 spider A. studiosus (mean FRP $=23.1)$, and 20-30.1 h (mean FRP = 24.5) in the southern black

90 widow Latrodectus mactans [13]. Even the orb weaver Metazygia wittfeldae (Araneidae), which

91 possesses an almost "typical" FRP (mean = 22.7 h) varied from $19-24$ hours [14]. The three

92 theridiid and two araneid species discussed here have FRPs that vary an order of magnitude more

93 than those of most animals examined thus far [13].

94 The high levels of FRP variance within each of these spider species sharply contrast with the

95 low levels of variance in other organisms, where it is suggested that the endogenous period of the

96 circadian clock is under tight genetic control $[11,15-18]$. The combination of high variation in

97 FRP and divergence of FRP from 24 hours suggests that spiders have evolved novel elements of

98 circadian control. Possible new mechanisms include a highly robust circadian response to light

99 stimuli, which allows rapid entrainment, or a weakly oscillating system that could be easily

100 perturbed by environmental stimuli, among others [13].

101 Thus far, the genetic control of circadian rhythms in spiders has not been investigated. To

102 identify genes potentially involved in circadian entrainment in spiders, we subjected the spider

103 species, $M$. wittefeldae, to two experimental conditions. In brief, we compared gene expression

104 between spiders kept in constant darkness to those exposed to a pulse of light. We used a

105 mathematical model to predict two time points with the greatest difference in gene expression 
106 levels between the constant dark group and the group exposed to a light pulse. Our simulations

107 further predicted that the direction of differential expression would switch between the two time

108 points. RNA-sequencing of four experimental groups of spiders identified a cluster of transcripts

109 that fit the prediction of a switched pattern of gene expression.

\section{Results}

\section{Mathematical model predicts optimal experimental protocol}

113 To optimize the search for circadian genes, we utilized a simplified mathematical model.

114 Most of the circadian clocks studied so far are based on a negative feedback loop, where a group

115 of circadian genes inhibit their own expression [2]. We therefore assumed that the circadian

116 clock in spiders has at its core at least one circadian gene (gene $X$ ). Translation of the circadian

117 gene's mRNA ( $m R N A X)$ induces production of the circadian protein (protein $X)$, which is

118 transferred back to the nucleus and inhibits its own transcription (Fig 1A). We simulated light in

119 the model by a rapid degradation of $m R N A X$. The model reproduces a wide range of free-

120 running periods in the dark as well as entrainment to LD 12:12.

121 Our experimental goal was to identify the light-sensitive subset of circadian genes. To

122 achieve this, we first entrained our model to LD 12:12 and then simulated a brief (1 hour) pulse

123 of light in progressively increasing time intervals after the end of the last light phase (pulse

124 group), while keeping an identical model in constant darkness (no pulse group) (Fig 1B). Based

125 on these simulations, we predicted that applying the light pulse 5 hours after the last light phase

126 (experimental time (et) 5 - et 6) would produce the greatest change in magnitude of gene

127 expression of the model's circadian $m R N A X$ (Fig 1B). To further improve the signal to noise

128 ratio, we also used the model to identify collection times that would provide the largest 
129 difference between the pulse and no pulse groups. By comparing the ratio of $m R N A X$ with and 130 without pulse at every hour after the light, we determined that collection times 7 and 16 hours

131 after the last light phase (et7 and et16), produces the largest difference between pulse and no 132 pulse conditions (Fig 1C).

(A)
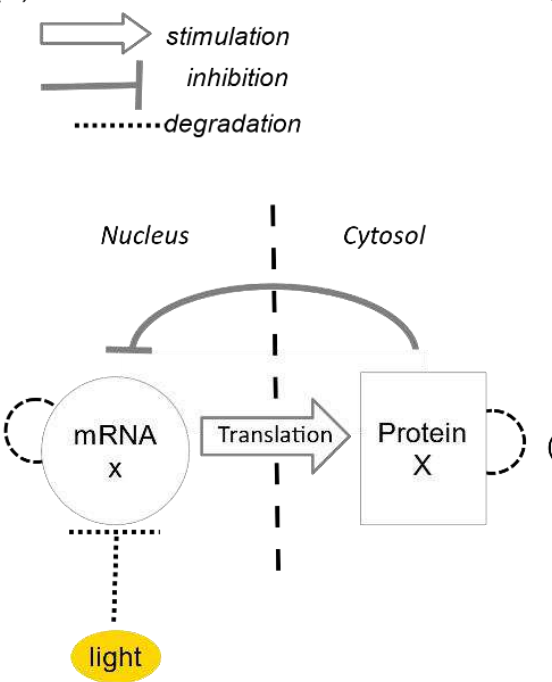

133

134 Figure 1: Modeling prediction leading to experimental design. (A) Mathematical model of circadian clock is based on hypothetical circadian mRNA inhibited by its own protein. (B)

136 Model simulation of 36 hours of LD 12:12 condition followed by DD conditions with (top) and 137 without (bottom) application of a brief (1 hr.) light pulse. The sampling times (red arrows in top 138 graph, blue arrows in bottom graph) were selected to optimize the differences between mRNA

139 levels with and without the light pulse. (C) Experimental protocol following the optimal

140 collection times predicted to yield the greatest differences in mRNA levels between the two 141 experimental conditions. 
143 Model predicts pattern of differential expression in response to light pulse and collection

144 times

145 Since light degrades circadian mRNA, the model predicts that one hour after light application

146 (et7), the group receiving a light pulse (pulse) will have significantly lower expression level than

147 the group receiving no light pulse (no pulse) (Fig 2A, filled bars). In other words, light induces a

148 phase advance (Fig 1B, compare top and bottom traces), where expression of circadian mRNA in

149 the pulse group lags behind the no pulse group. As a result of this phase advance, at the time of

150 the second collection (et16), the pattern of mRNA expression reverses, with the pulse group

151 having a much higher expression level than the no pulse group (Fig 2A, open bars). The reason

152 for the flipped mRNA expression at et16 is that the no pulse group reaches its trough while the

153 lagging pulse group is almost at its peak.

154 Since we used our model to predict the pattern of differential expression of circadian light-

155 sensitive genes, we can compare the ratio (also called Fold Change) of pulse vs. no pulse groups

156 at two collection time points (Fig 2C). At et7, expression in the pulse group is lower than in the

157 no pulse group, resulting in negative log Fold Change (log FC). But at et16, expression in the

158 pulse group exceeds the no pulse group, resulting in a positive log FC. We therefore expect the

159 circadian genes in our experiment to reverse the log FC values.

160 Our model also predicts similar reverse pattern of differential expression in response to

161 collection times when comparing the pulse to no pulse groups. In the pulse group, mRNA

162 expression is much higher at et16 than et7 (Fig 2B, red bars), generating a negative log FC

163 (et7/et16) (Fig 2 D, left). In the no pulse group, which did not experience mRNA suppression,

164 the relationship is opposite, with mRNA level higher at et7 than et16 (Fig 2B, blue boxes). In

165 other words, the differential expression with respect to collection time in no pulse group 
167 expression of circadian genes will be reversed by both pulse and collection time conditions.

(A)

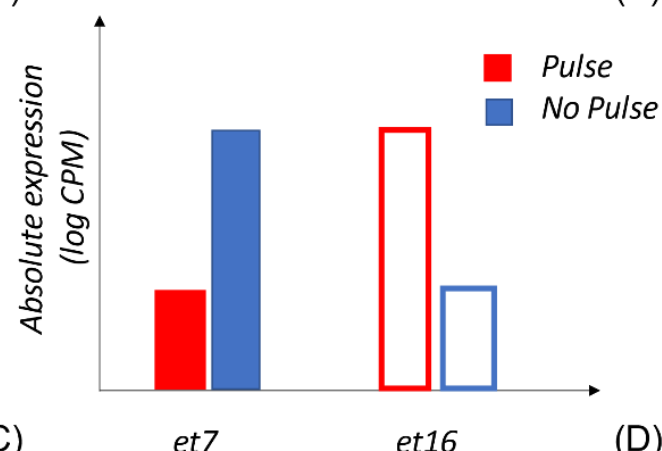

(C)

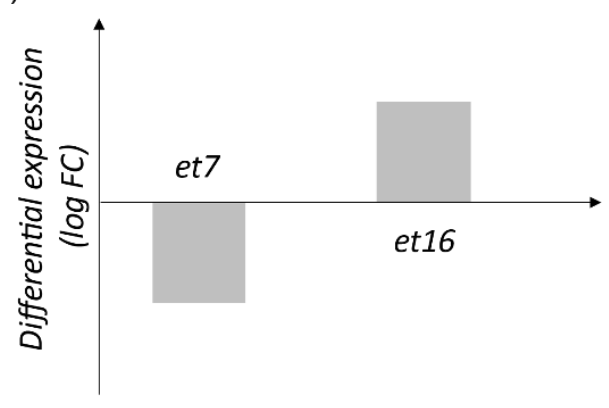

(B)

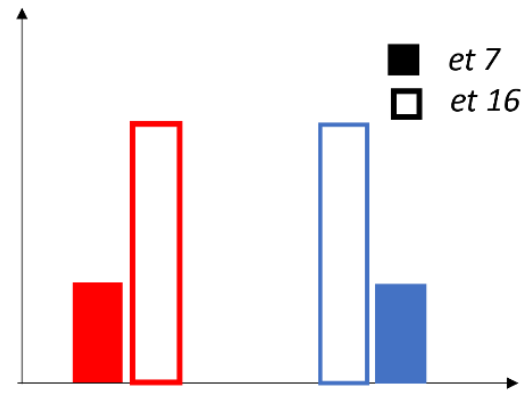

(D)

Pulse No Pulse

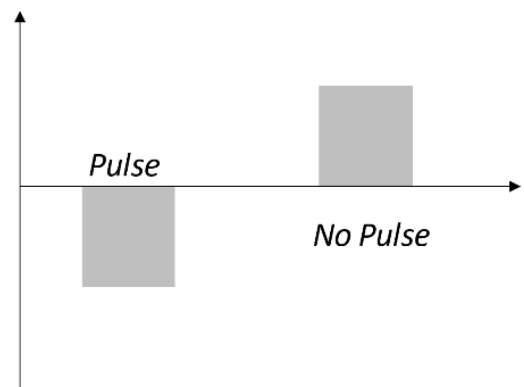

171 Figure 2: Expected outcomes of experimental protocol predicted by the model for light-sensitive

172 genes. (A) Absolute gene expression is expected to be increased by the 1-hour pulse of light

173 (pulse) in et7, but decreased at et16. (B) Absolute gene expression with the pulse is higher at

174 et16 compared with et11. Without the pulse of light (no pulse), the trend is opposite, with higher

175 expression at et16. (C) Differential expression, measured as log of ratio (log FC) between pulse

176 and no pulse condition is negative in et7 and positive at et16. (D) Differential expression,

177 measured as $\log \mathrm{FC}$ between et7 and et16 times is negative in pulse condition and positive in no 178 pulse condition. 
181 We used our model to optimize the experimental timeline for spider entrainment, application of

182 the light pulse, and tissue collection from the cephalothoraxes of adult female $M$. wittfeldae.

183 These experimental spiders included 10 in the pulse and 10 in the no pulse groups. The pulse

184 group received a 1-hour light pulse five hours (et5-6) after lights-off at the end of the $5^{\text {th }}$ day of

185 LD 12:12 but no light thereafter. The no pulse group received no light after the $5^{\text {th }}$ day of LD

186 12:12. In each group, 5 spiders were sacrificed in the dark 7 hours (et7) and 16 hours (et 16) after

187 the last LD 12:12 lights-off transition (see Figure 1C).

188 We de novo assembled 266,300 transcripts from the 20 individual RNA-seq libraries,

189 representing 184,077 Trinity-defined "genes". Annotation of the "genes" resulted in 9 categories

190 of sequences, 4 of which were unlikely to encode proteins and were thus removed prior to further

191 analyses (Table 1). Of the retained translated genes, 25,489 were further annotated by significant

192 alignment to a protein in SwissProt and/or PFAM, and 25,127 were assigned GO Terms based on

193 the SwissProt, PFAM, and/or D. melanogaster alignments. Comparison with 1066

194 Benchmarking Universal Single Copy Orthologs (BUSCO v3.0.2, [19]) from arthropods

195 suggests that our transcriptome is of high quality, with $98.4 \%$ (91\% as single copy and $7.4 \%$ as

196 duplicates) of BUSCO genes represented completely and 0.3\% represented by fragmented

197 sequences.

198

Table 1. Annotation of the de novo assembly of Metazygia wittfeldae.

\begin{tabular}{|l|l|}
\hline BEST: has a reciprocal best BLASTx alignment to a protein in a reference database & 26114 \\
\hline GOOD: has BLASTx alignment to a protein in one of the reference databases but & 9655 \\
another Trinity gene has a better BLAST alignment & \\
\hline
\end{tabular}




\begin{tabular}{|c|c|}
\hline $\begin{array}{l}\text { LONGORF: predicted to encode a protein at least } 50 \text { amino acid long, but does not } \\
\text { have a significant BLASTx alignment to any of the reference proteins }\end{array}$ & 46325 \\
\hline LOW_EXP: same as GOOD, but with $<1$ RSEM estimated TPM in any library & 10953 \\
\hline $\begin{array}{l}\text { LOW_EXP_LONGORF: same as LONGORF, but with }<1 \text { RSEM estimated TPM } \\
\text { in any library }\end{array}$ & 42737 \\
\hline $\begin{array}{l}\text { CHIMERA: potential assembly of two different protein coding genes because of a } \\
\text { very good alignment to different proteins in the reference databases }\end{array}$ & 48 \\
\hline $\begin{array}{l}\text { LOW_COV: same as GOOD, except that it only aligns to }<20 \% \text { of the database } \\
\text { protein }\end{array}$ & 7881 \\
\hline RNA: aligns to ribosomal or transfer RNA & 210 \\
\hline $\begin{array}{l}\text { NO_HIT: has no BLAST alignment and is not predicted to encode a protein }>50 \\
\text { amino acids }\end{array}$ & 40153 \\
\hline \multicolumn{2}{|l|}{$\begin{array}{l}\text { Counts are based on the Trinity-defined "gene". The bottom } 4 \text { categories were retained for } \\
\text { read mapping and estimation of expression levels with RSEM, but were removed prior to } \\
\text { differential expression analyses (see details in Methods). TPM = RSEM [20] estimated }\end{array}$} \\
\hline
\end{tabular}

200 We conducted four separate differential expression analyses at the "gene" level (see Methods 201 and Additional File 1: Table S1). MDS (Multidimensional scaling) plots (Additional File 2:

202 Figure S1) illustrate the relative clustering of samples in different conditions. A light pulse in the 203 middle of the night (et5-et6) resulted in individuals having the most similar gene expression 204 profiles of any of the four experimental conditions (Additional File 2: Figure S1). The pulse of 205 light also resulted in many more differentially expressed genes 1-hour after the light pulse than 
10 hours later (282 vs. 57, Table 2, Figure 3). Consistent with a large effect of light on gene

207 expression, more genes were differentially expressed between the two collection times in the

208 group that received a light pulse than the one that did not (227 vs. 95, Table 2, Figure 3 ). The

209 light pulse caused many more genes to be downregulated (70\%) than upregulated (30\%) relative

210 to no light pulse, when collected at et7 (Additional File 2: Figure S2); however, 10 hours later,

211 approximately equal number of DE genes were upregulated and downregulated (Table 2 and

212 Additional File 2: Figure S2). These findings are consistent with the prediction of our model that

213 light would degrade circadian transcripts, but that expression would subsequently increase over

214 time (Figure 1b).

215

Table 2. Differentially expressed genes.

\begin{tabular}{|l|l|l|}
\hline Comparison & \# DE genes (FDR < 0.05) & \# genes examined \\
\hline pulse vs. no pulse at et7 & $282(211$ down, 71 up) & 90341 \\
\hline pulse vs. no pulse at et16 & $57(30$ down, 27 up) & 102817 \\
\hline et7 vs. et16 with pulse & $227(165$ down, 62 up) & 100103 \\
\hline et7 vs. et16 with no pulse & $95(52$ down, 43 up) & 94062 \\
\hline
\end{tabular}

Four comparisons were performed (Additional file 1: Table S1) to identify differentially

expressed genes (false discovery rate, FDR $<0.05$, $\mid$ log fold change $\mid>1$ ) with edgeR (Robinson and Oshlack 2010), focusing only on genes with > 0.1 CPM in at least two libraries. Spiders received a 1-hour pulse of light 5 hours after lights off (pulse) or did not (no pulse) and were collected 1 hour (et7) or 10 hours (et16) after the end of the light pulse. 
Despite a large effect of the 1-hour light pulse on differential expression patterns (Table 2),

218 we found limited overlap in the identities of differentially expressed genes among comparisons

219 (Figure 3). The greatest overlap was between pulse vs. no pulse at et7 and et7 vs. et16 with

220 pulse. The 93 genes that were differentially expressed in both comparisons are strong candidates

221 for light responsive circadian genes.

222

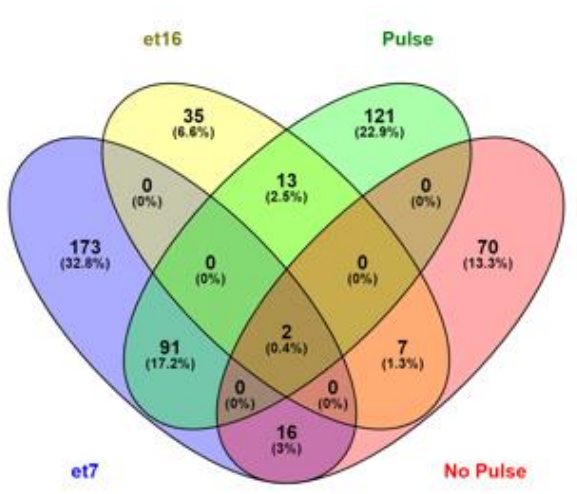

Figure 3: Venn diagram shows unique and shared DE genes in response to light pulse and collection time. The intersections between colors show the number of DE genes in common to different treatments. et7: Differentially expressed transcripts with light pulse vs without light pulse

229 at et7; et16: Differentially expressed transcripts with light pulse vs without light pulse at et16;

230 Pulse: Differentially expressed transcripts at et7 vs et16 with light pulse; No Pulse: Differentially

231 expressed transcripts at et7 vs et16 without light pulse.

233 Canonical clock genes are lowly and weakly differentially expressed

234 We also investigated the expression patterns of 7 genes known to control the circadian clock in 235 the fruit fly and other arthropods. We identified orthologs of each of these 7 canonical clock 236 genes (tim, per, cyc, Clk, dbt, cry1, cry2) in our $M$. wittfeldae transcriptome using a phylogenetic 237 approach (see Methods for details). Of the 7, only cry2 was found to be significantly

238 differentially expressed (FDR < 0.01). Specifically, cry2 was more highly expressed at the 239 second collection time (et16) than the first (et7) when not subject to a light pulse (Figure 4a). Clk 
240 had a similar pattern of expression as cry2, although none of the comparisons met the FDR

241 threshold for significance (Figure 4a). In addition, $c l k$, cry2, and $c y c$ have significant positively

242 correlated pattern of expression when considering expression levels of all four conditions (Figure

243 4b). Cyc was also significantly correlated with $d b t$ (Figure 4b). However, $c y c$ and tim were very

244 lowly expressed ( $<1$ TPM in all individuals, Additional File 2, Figure S3).

245 Despite minimal differential expression of clock genes among our conditions, each clock

246 gene was significantly correlated with numerous DE genes (Cyc: 51, Clk: 71, Dbt: 81, Per: 39,

247 Tim: 32, Cry1: 54, Cry2: 75; Additional File 3, Table S2). The functions of DE genes that

248 significantly correlated with at least one clock gene include transcriptional regulators,

249 metabolism regulation, protein modifications, and embryonic development, among other

250 functions (Additional File 3, Table S2).

251

252

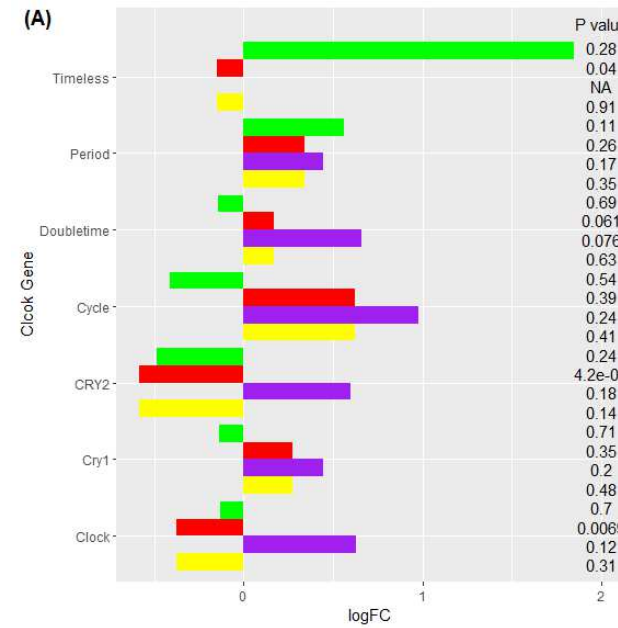

(B)

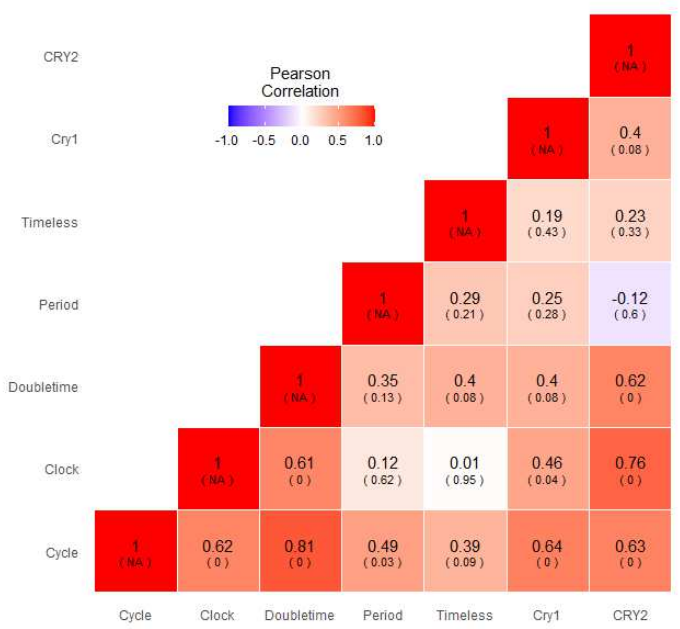

253 Figure 4: Expression patterns of seven canonical clock genes. (A) Log FC values of the seven

254 canonical clock genes with corresponding P-values (from edgeR [21]) rounded to two significant

255 digits in response to collection time and light pulse. (B) Correlation coefficients among the seven

256 canonical clock genes. Positive correlation coefficients are colored red while negative values are 
258 digits.

\section{Light elicits a variety of functional changes}

260 To determine the functional significance of transcripts differentially expressed in our

261 experimental conditions, we compared Gene Ontology (GO) terms associated with the DE genes

262 to GO terms assigned to the entire transcriptome (see Methods). Once we identified GO terms

263 enriched in DE genes (Additional File 4: Table S3), we used the REVIGO reduction analysis tool

264 to visualize their functions (e.g. Figure 5). The pulse of light produced more enriched GO Terms

265 between time points than the constant dark condition (Figure 5, compare C and D). When

266 comparing pulse to no pulse conditions, the earlier collection time led to more enriched GO

267 terms (Figure 5, compare A and B). Comparison of the pulse to no pulse conditions at the early

268 collection time, et 7 (Figure 5A), yielded enrichment of GO-terms in Biological Process (BP)

269 category related to cell death and response to stress. The enriched GO terms suggest that the light

270 pulse affected developmental processes including vesicle-mediated transport, embryo

271 development, and cell division (Additional File 4: Table S3). Also, some regulatory processes

272 were likely modified based on the enriched GO terms membrane organization, vesicle-mediated

273 transport, protein targeting and cytoskeleton organization. Analysis of enriched GO terms in the

274 Cellular Component (CC) category demonstrates that gene expression changes were distributed

275 within the cell, because components included lysosome, Golgi apparatus, nuclear chromosome,

276 and extracellular region. Finally, analysis of Molecular Function (MF) category resulted in

277 enriched GO-terms in structural molecule activity and GTPase activity (Additional File 4: Table

278 S3). 
In contrast, comparing the pulse to no pulse conditions at the late collection point, et16

280 (Figure 4B), there are only 2 enriched GO-terms in the BP category: cell adhesion and response

281 to stress. The only molecular function enriched was in structural molecule activity (Additional

282 File 4: Table S3).

283 When comparing the two collection times, the pulse condition yielded the largest pool of

284 enriched GO terms (Figure 5C). Our analysis indicates that a wide range of biological processes

285 were affected. Notably, response to stress and cell death were among the enriched GO terms.

286 Also, GO terms related to proliferations were enriched, such as growth, embryo development,

287 and cell differentiation, among others. In addition, enriched GO-terms in this category included

288 some of the regulatory processes such as translation, secondary metabolic processes, etc. Those

289 processes were not localized, but widely distributed within and outside the cell, since cellular

290 component enriched terms included cell, organelle, nuclear chromosome and extracellular space.

291 However, only three molecular functions were enriched: GTPase activity, oxidoreductase

292 activity, unfolded protein binding (Additional File 4: Table S3).

293 Finally, when comparing the two collection times, the no pulse condition yielded only a few

294 enriched terms (Figure 5D). Biological processes included carbohydrate metabolic processes,

295 cell population proliferation, and aging. However, this effect was likely localized to extracellular

296 space because it was the only enriched group in the CC category. In the molecular function

297 category, terms involved ion binding, enzyme regulator activity, and hydrolase activity acting on

298 glycosyl bonds were enriched (Additional File 4: Table S3). 


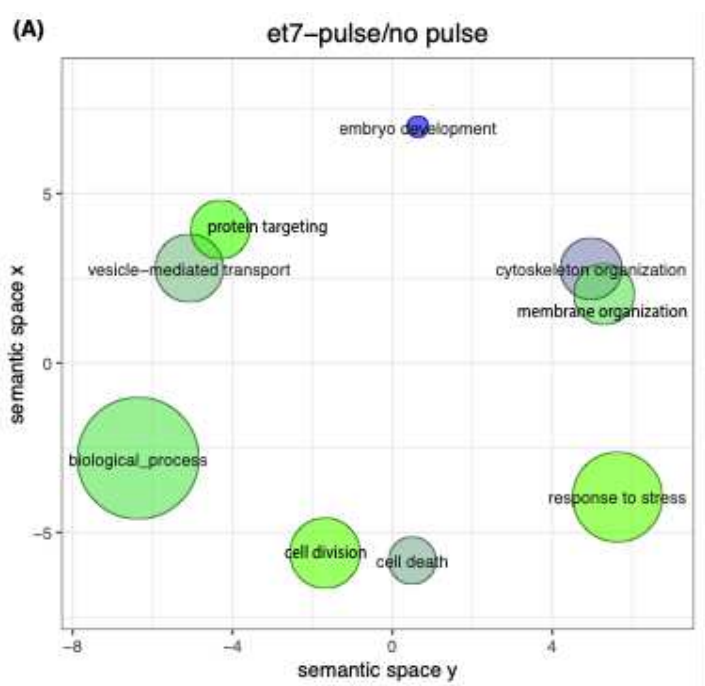

(C)

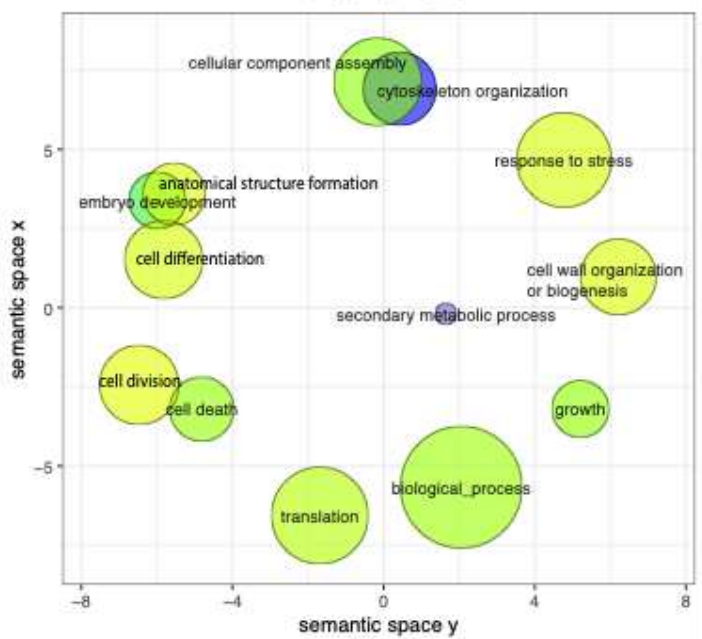

(B)

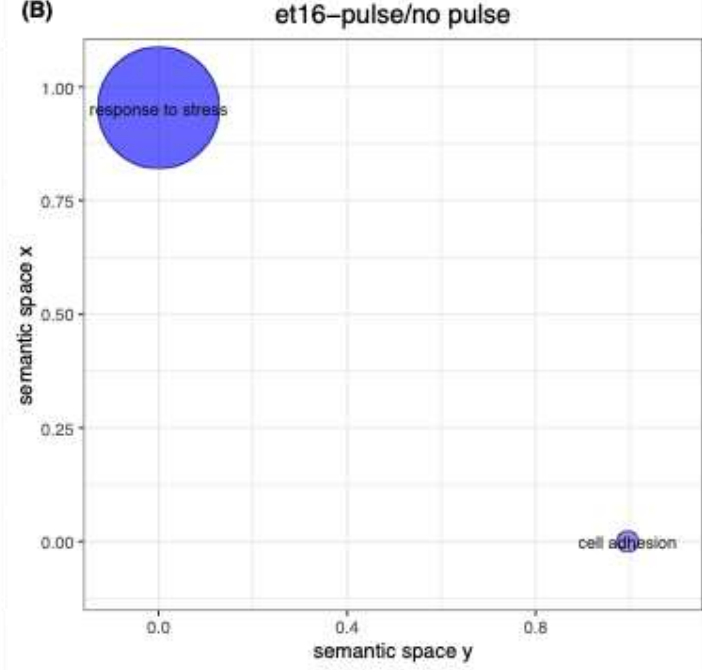

(D)

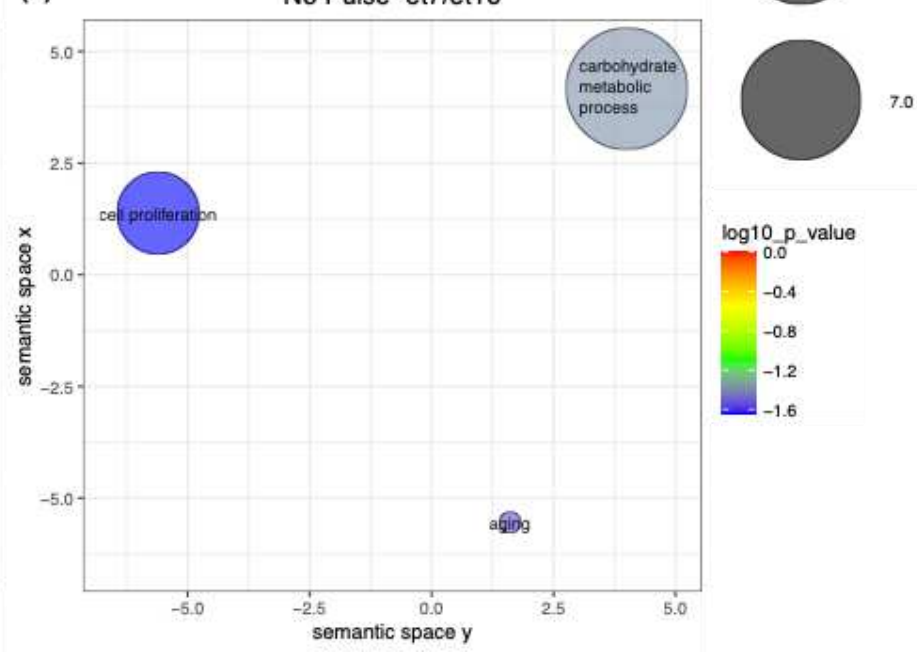

Figure 5: REVIGO scatterplots of the enriched GO cluster representatives related to biological

302 processes. The resulting lists of GO terms (Additional file 4: Table S3) along with their p-values

303 were summarized and visualized by REVIGO reduction analysis tool. (A) Significantly enriched

304 GO terms related to biological processes with light pulse vs without light pulse at et7. (B)

305 Significantly enriched GO terms related to biological processes with light pulse vs without light

306 pulse at et16. (C) Significantly enriched GO terms related to biological processes at et7 vs et16

307 with light pulse. (D) Significantly enriched GO terms related to biological processes at et7 vs

308 et16 without light pulse. GO terms are represented by bubbles and are clustered according to

309 their semantic similarities to other GO terms in the gene ontology (adjoining circles are most 
311 colors are red and blue, depicting lower- and higher p-values respectively. Bubble size indicates

312 the frequency of the GO term in the underlying reference EBI GOA database [22] (bubbles of

313 more general terms are larger).

\section{Flipped patterns of expression predicted by the model identifies numerous circadian genes}

316 Our model predicts that circadian genes follow a well-defined pattern of expression in response

317 to light pulse and collection time. Specifically, at et7 circadian genes should be downregulated

318 by a pulse of light compared to the no pulse condition (Figure 2A, left). Circadian gene

319 expression is expected to be flipped at et16, where they become upregulated by light (Figure 2A,

320 right). As a result, we expected the ratio of mRNA expression between pulse and no pulse

321 conditions (log FC) to reverse between the two collection times, with negative log FC at et7 and

322 positive $\log \mathrm{FC}$ at et16 (Figure 2C). To visualize this prediction, we introduced a new coordinate

323 system where $\log \mathrm{FC}$ at et7 is plotted on the horizontal axis and log FC at et16 is plotted on the

324 vertical axis. Our model predicts that $\log \mathrm{FC}$ at et7 would be negative, thus circadian genes

325 would be located at the left plane. Another prediction of the model is that log FC at et17 would

326 be positive and, therefore, circadian genes would be located on the upper half plane. In summary,

327 our model predicts that, in this coordinate system, circadian light-sensitive genes will be in the

328 upper left quadrant. When we plotted our experimentally determined DE transcripts, we found a

329 large cluster of DE transcripts in the upper left quadrant, in the location predicted by our model

330 (Figure 6A; Additional File 5: Table S4). Therefore, we hypothesize that the transcripts in the

331 upper left quadrant of figure 6A are involved in the circadian response to light. 
Similarly, our model predicts that circadian genes should be downregulated at et7 compared

333 to et16 in the pulse condition (Figure 2B, left). Circadian gene expression is expected to be

334 flipped in the no pulse condition (Figure 2B, right). As a result, we expect a negative log FC with

335 a pulse and a positive log FC with no pulse (Figure 2D). In our coordinate system that plots log

336 FC in pulse condition on the horizontal axis, downregulated circadian transcripts would be on the

337 left-half plane. If log FC in no pulse condition is plotted on the vertical axis, the upregulated

338 transcripts would be on the upper half-plane. Like collection time analysis, the circadian light-

339 sensitive genes should be located at the upper left quadrant. Our experimentally determined DE

340 genes in fact show a large cluster of transcripts in the upper left quadrant, where the model

341 predicts our circadian light-sensitive genes would be located (Figure 6B, Additional File 5: Table

342 S4). Therefore, we hypothesize that the transcripts in the third quadrant of figure $5 \mathrm{~B}$ are involved

343 in circadian response to a pulse of light.

344 Our proposed light-sensitive circadian genes (upper left quadrants of Figure 6A\&B) are

345 enriched for a number of GO terms identified in the 4 separate comparisons (Figure 4) as well as

346 additional GO terms (Table 3). The similar pattern of expression found for these proposed light-

347 sensitive circadian genes is further supported by the fact that the majority of transcripts in each

348 quadrant are significantly correlated with each other when considering all four experimental

349 conditions (56\% of the 140 genes in upper left quadrant of Figure $6 \mathrm{~A} ; 68 \%$ of the 170 genes in

350 upper left quadrant of Figure 6B). 

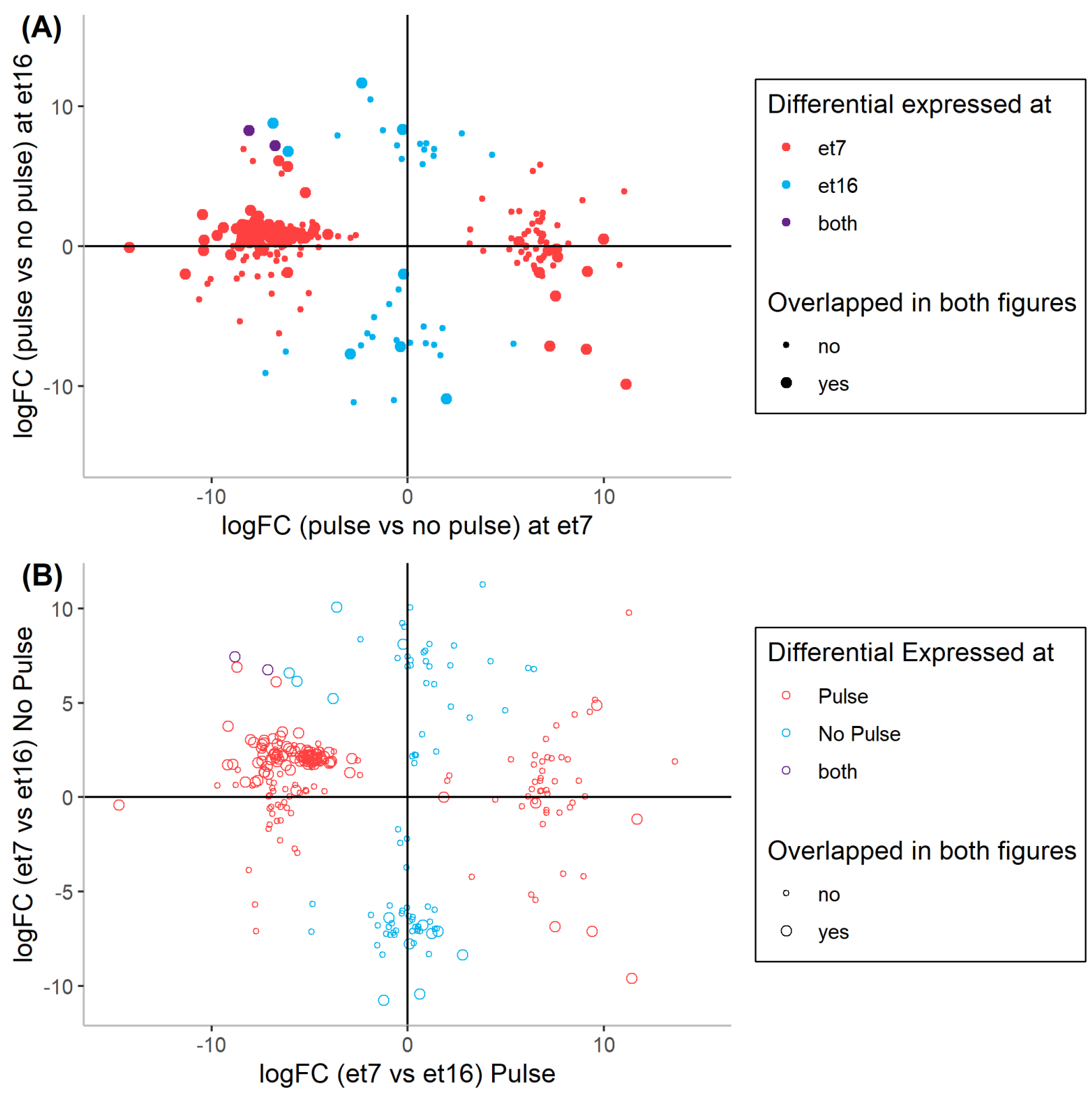
Differential Expressed at
- Pulse
- No Pulse
$\circ$ both
Overlapped in both figures
- no
$\circ$ yes

353 Figure 6: Cluster of transcripts in response to collection time and light pulse. Circles in

354 scatterplots indicate log FC values of differentially expressed genes (FDR < 0.05, $|\log \mathrm{FC}|>1$ ).

355 (A) Top left corner contains the cluster of genes whose log of ratio (log FC) values between

356 pulse and no pulse condition are negative in et7 and positive at et16 as predicted by the model

357 (Figure 2 A \& C). Transcripts differentially expressed between pulse and no pulse at only et7 are 358 red filled dots while genes differentially expressed at only et16 are colored blue. One gene is 
359 differentially expressed at both et7 and et16, colored purple. (B) Top left corner contains the

360 cluster of genes whose log FC values between et7 and et16 times are negative in pulse condition

361 and positive in no pulse condition as predicted by the model (Figure 2 B \& D). Transcripts

362 differentially expressed only with light pulse are open circles while transcripts differentially

363 expressed only without light pulse are filled circles. One gene is differentially expressed with or

364 without light pulse, colored purple. The larger circles indicate genes identified in both A \& B.

Table 3. GO terms enriched in the circadian light-responsive transcripts. 


\begin{tabular}{|c|c|c|}
\hline $\begin{array}{l}\text { Cellular } \\
\text { Component }\end{array}$ & $\begin{array}{l}\text { - } \\
\text { - } \quad \text { extracellular region } \\
\text { - lysosome }\end{array}$ & $\begin{array}{l}\text { - } \quad \text { Golgi apparatus } \\
\text { - } \quad \text { plasma membrane } \\
\text { - } \quad \text { endoplasmic reticulum }\end{array}$ \\
\hline $\begin{array}{l}\text { Biological } \\
\text { Process }\end{array}$ & $\begin{array}{l}\text { - } \text { signal transduction } \\
\text { - } \text { anatomical structure development } \\
\text { - } \quad \text { cell division } \\
\text { - } \quad \text { cell morphogenesis } \\
\text { - } \text { transport } \\
\text { - } \quad \text { cell junction organization } \\
\text { - } \quad \text { reproduction } \\
\text { - } \quad \text { vacuolar transport } \\
\text { - } \quad \text { anatomical structure formation } \\
\text { - } \quad \text { involved in morphogenesis } \\
\text { - } \text { cell motility }\end{array}$ & $\begin{array}{l}\text { - } \text { cell population proliferation } \\
\text { - } \text { plasma membrane organization } \\
\text { - } \text { membrane organization } \\
\text { - } \text { secondary metabolic process } \\
\text { - } \text { cell differentiation } \\
\text { - } \text { cell death } \\
\text { - growth } \\
\text { - } \text { protein folding } \\
\text { - } \text { vesicle-mediated transport } \\
\text { - } \text { response to stress } \\
\text { - } \text { embryo development } \\
\text { - } \text { cytoskeleton organization }\end{array}$ \\
\hline Molecular & $\begin{array}{ll}\text { - } & \text { unfolded protein binding } \\
\text { - } & \text { structural molecule activity } \\
\text { - } & \text { GTPase activity }\end{array}$ & \\
\hline \multicolumn{3}{|c|}{$\begin{array}{l}\text { Light-responsive circadian transcripts are those with a flipped pattern of expression between time points } \\
\text { depending on the light experiment (upper left quadrants of Fig. } 6 \text { A\&B). The GO terms in this table are enriched } \\
\text { in these transcripts relative to the entire transcriptome. }\end{array}$} \\
\hline
\end{tabular}

\section{Discussion}

368 To our knowledge, this study is the first to investigate genetic mechanisms of the circadian

369 clock in spiders. By comparing differential gene expression between two time points under two 
370 light conditions, we were able to identify potential cycling genes, as well as candidate genes for 371 circadian light entrainment.

372 The total number of DE transcripts between our two collection times (227 for pulse, 95 for 373 no pulse, 2 overlap, Fig. 3) is consistent with previously reported sets of time-dependent $D$.

374 melanogaster genes. However, our experimental application of a 1-hour light pulse in the middle 375 of the night is unusual. For example, a cDNA chip experiment of 13,500 genes in $D$.

376 melanogaster identified 120 cycling transcripts by sampling every 4 hours for 2 days in a 12:12

377 LD cycle; approximately 50\% of these also cycled in constant darkness [23]. A GeneChip

378 (13,232 unique probes) analysis identified many more genes, 712 , that showed a daily fluctuation 379 in mRNA levels under light-dark conditions; 115 of which also cycled in DD [24]. High-density 380 oligonucleotide arrays identified 400 oscillating transcripts in DD [25]. A meta-analysis of 381 these 3 studies and 2 additional microarray based studies found 218 significantly cycling 382 transcripts, combining LD and DD conditions [26]. We identified homologs of 80 of these $D$. 383 melanogaster cycling transcripts in our $M$. wittfeldae transcriptome but the spider homologs 384 were not differentially expressed between our collection times or between the light pulse and no 385 pulse conditions. The lack of overlap in differentially expressed genes between $D$. melanogaster 386 and M. wittfeldae suggests that the circadian mechanism, or at least the circadian output, in 387 spiders differs significantly from the fruit fly. The idea of distinct set of circadian genes in 388 spiders was also supported by the finding that a large fraction of DE genes in our data set did not 389 have homologs outside of Araneae (75\% of the 385 genes DE between time points).

390 Our comparison of two time points in constant darkness is most similar to the $D$.

391 melanogaster experiments described above. Only 95 M. wittfeldae transcripts were differentially 392 expressed under these conditions, which is similar, slightly lower, or considerably lower than the 
393 number reported for D. melanogaster in constant dark, depending on the study (see previous

394 paragraph). Several potential explanations can be offered to justify the smaller number of DE

395 transcripts in our experiment. Although we used a mathematical model to select collection time

396 points to amplify the circadian signal, the lack of experimental data on kinetic constants for

397 circadian mRNA and protein might result in some missing transcripts. For example, the

398 transcripts in the middle of the rising and falling phases would have a similar level of expression

399 in our experimental conditions (Fig. 1). In the future, more frequent sampling (at least every 4

400 hours) should be conducted to identify all cycling transcripts. Nevertheless, we identified a

401 sizable cluster of DE transcripts with the flipped pattern of expression predicted by our model

402 (Figure 6). We thus feel confident that we have captured many light-sensitive circadian genes,

403 even if we have not captured all the cycling transcripts.

404 An alternative explanation of the small number of DE genes between time points might be an

405 overall lower level of circadian gene expression in spiders. It is possible that the circadian

406 oscillator in spiders has a lower amplitude than other species. Based on simulations with our

407 model, and prior evidence [27-30], we hypothesized that such weaker circadian clock could

408 explain an unusually wide range of free-running periods observed in spiders [13]. It is therefore

409 possible that some evolutionary adaptation in spiders leads to lower level of circadian gene

410 expression.

411 Another line of evidence that could support the weak oscillator model is the very low

412 expression levels of the canonical clock genes, tim and cyc, in all conditions. The degree of

413 difference between time points for 6 of the 7 canonical clock genes examined was also low

414 (Figure 4A), consistent with low amplitude oscillations. Only cry2 met our criteria for

415 significantly different expression levels, and only between the two time points in constant 
416 darkness. Cry2 is a member of the photo-lyase gene family, but its protein product,

417 cryptochrome-2 is not light-sensitive [8]. In multiple arthropod species, CRY2 inhibits

418 CLOCK:CYCLE-mediated transcription [8,9]. We would thus predict a negative correlation

419 with per and tim (e.g. as in mosquitoes, [31]). We found a weak negative correlation between

$420 \quad$ cry2 and per but a weak positive correlation with tim. On the other hand, cry 2 was significantly

421 positively correlated with $c l k$ and $c y c$ (Figure 4B), consistent with a possible interaction among

422 their protein products. We also found that the clock genes were significantly correlated with

423 genes encoding functions often under circadian control. It is thus possible that despite low

424 amplitude differences, and unexpected correlations with each other, the canonical clock genes

425 are important for circadian control in spiders. This control, however, may differ dramatically

426 from what has been previously described.

428 Short term response to light pulse produces largest set of DE transcripts

429 By comparing differential gene expression patterns between pulse and no pulse groups in our

430 experiment, we identified transcripts involved in short- and long-term responses to light. We

431 found a larger number of genes were DE at the earlier collection time than the latter, suggesting

432 an acute short-term response to the light pulse. Interestingly, a sizable portion of those light-

433 responsive transcripts encode proteins with a wide range of cellular functions. Those results are

434 consistent with previous findings in other species. For example, [32] compared response to 1 and

4353 hours of 1,200 lux light pulse in zebra fish. They identified 117 light-regulated genes, with the

436 majority being induced and some repressed by light. The regulated genes cover a variety of

437 functions including stress response and DNA repair, consistent with our findings. 
Another study measured response of the Arabidopsis transcriptome to 1-hour light pulses

439 given either in the middle of the subjective day or in the middle of the subjective night [33]. This

440 study identified a total of 2,237 light-sensitive genes, which is much higher than in our study. A

441 light stimulus during the night preferentially promoted the expression of certain key clock

442 components, consistent with the general observation that light present at the beginning or end of

443 the photoperiod adjusts the circadian clock to seasonal changes in day length.

444 We were unable to find gene expression studies exposing any arthropod species to a short

445 pulse of light. The most comparable arthropod experiments we could find applied weak light at

446 night. For example, fireflies exposed to weak light at night for 2 weeks experienced much higher

447 mortality than fireflies not exposed to light at night [34]. Furthermore, weak light at night

448 resulted in 1262 down regulated and 105 upregulated genes [34]. We similarly found more

449 downregulated genes in the short-term response to a light pulse in M. wittfeldae. Enriched GO

450 terms in the firefly DE genes included functions related to immune response, development,

451 reproduction, and biological rhythm. Some of the immune response and development terms

452 reflect GO terms we found with response to light. The DE genes related to biological rhythm

453 were not the "main" clock genes but ones that interact with the "main" clock - so it is perhaps

454 not surprising that we did not identify an effect of light on canonical clock genes.

Transcripts involved in the circadian response to light relate to immune function, growth,

\section{7 development, and reproduction}

458 Our most striking finding is the cluster of transcripts with switched patterns of differential 459 expression over time, dependent on light pulse, as predicted by our mathematical model (Fig. 6).

460 These transcripts represent our best candidates for light-sensitive circadian genes. These 
transcripts had multiple functions and were distributed throughout the cell (Table 3). GO-terms

462 involved in immune response, growth, development, and reproduction were enriched in this

463 candidate set of light-sensitive circadian genes (Table 3). It has been shown in D. melanogaster

464 that the immune response is under circadian control $[35,36]$. It is also has been found that

465 exposure to night light leads to reductions in immune function in vertebrates and invertebrates

$466[37,38]$.

467 We also found enriched GO-terms for growth, development, and reproduction in our

468 candidate light-sensitive circadian genes, such as "cell morphogenesis", "embryo development",

469 “cell differentiation", and "cell division" (Table 3). These results are consistent with prior

470 evidence that exposure to light at night alters circadian rhythms, inhibits reproduction,

471 accelerates development, and leads to increased mortality (e.g. vertebrates [37], fireflies [34],

472 and D. melanogaster [39]). In an Australian nocturnal orb weaving spider, exposure to artificial

473 light at night accelerated juvenile development, resulting in spiders progressing through fewer

474 molts [40]. Light intensity also controls the eclosion circadian rhythm in D. melanogaster [41].

475 Our results for $M$. wittfeldae further support a role of light in affecting the circadian rhythms of

476 immune function, development, and reproduction. Our transcripts with flipped patterns of

477 expression between time points, dependent on light (Fig. 6) may even include ones important for

478 entraining the circadian output to light.

\section{Conclusions}

481 To our knowledge, this work is the first to identify circadian and light-responsive transcripts in

482 spiders. We used a mathematical model of the circadian clock in spiders to design a light

483 application experiment, and the subsequent collection times to optimize differences in gene 
484 expression. By comparing the levels of expression at two time points after an application of light,

485 or not, we were able to identify distinct clusters of transcripts responding to time of day and

486 light. These transcripts are our best candidate set for light-sensitive circadian genes. Many of

487 these transcripts likely encode outputs of an entrained circadian clock, including immune

488 response, reproduction, and development. However, it also is possible that some of these

489 transcripts encode central components of the clock that are critical to entrainment. Given the

490 unusual characteristics of spider free-running periods, and the fact that many of our candidate

491 light-sensitive circadian genes have no homologs outside of spiders, spiders may have evolved

492 unique genetic mechanisms of entrainment and intrinsic circadian cycling.

\section{Methods}

\section{Mathematical Model}

496 Briefly, the minimal model of spider circadian clock was based on a previously developed

497 Neurospora model [42] that involves a feedback loop among (i) a specific mRNA, (ii) the

498 cytosolic protein encoded by this mRNA, and (iii) the gradual phosphorylation of the cytosolic

499 protein, which changes its structure, enabling it to enter the nucleus. As illustrated in Figure 1(a),

500 the mRNA, produced from a gene through the process of transcription, is positively coupled to

501 the cytosolic protein through the process of translation. After the cytosolic protein changes its

502 structure and re-enters the nucleus, the resulting nuclear protein acts to suppress expression of

503 the gene coding for the mRNA, representing a negative coupling. Natural degradation of the

504 mRNA sequence and the cytosolic protein is also included in the model.

505 The coupled system of equations governing the circadian oscillator is as follows:

$506 \frac{d M}{d t}=v_{s} \frac{K_{1}^{2}}{K_{1}^{2}+P_{N}^{n}}-v_{m} \frac{M}{K_{m}+M}-L M$ 
$507 \frac{d P_{c}}{d t}=k_{s} M-v_{d} \frac{P_{C}}{K_{d}+P_{C}}-k_{1} P_{C}+k_{2} P_{N}$

$508 \quad \frac{d P_{N}}{d t}=k_{1} P_{C}-k_{2} P_{N}$

510 The three variables $M, P_{C}$, and $P_{N}$ represent the concentrations (with respect to cell volume)

511 of the mRNA, the cytosolic protein, and the nuclear protein, respectively. The parameters of the

512 are minimally modified from the original model [42]. The rate of mRNA production is inhibited

513 by nuclear protein, $P_{N}$, with second order rate parameters $K_{1}=1 n M^{1 / 2}, v_{s}=1.6 \frac{n M}{h r}$. mRNA

514 also degrades with a first order rate parameters $v_{m}=0.5 \frac{n M}{h r}, K_{m}=0.5 n M$. Exposure to light

515 induces additional, exponential rate of mRNA degradation with parameter $L=h r^{-1}$. mRNA is

516 translated into protein with effective rate $k_{s}=0.5 \mathrm{hr}^{-1}$, undergoes first order degradation with

517 parameters $v_{d}=1.4 \frac{n M}{h r}, K_{d}=0.15 n M$, and is transported to nucleus and back with linear

518 rates respectively $k_{1}=0.5 h r^{-1}, k_{2}=0.6 h r^{-1}$.

\section{Experimental Setup}

521 We used the model to predict the time to apply a 1-hour light pulse that would maximize the

522 difference in gene expression levels between spiders receiving the light pulse and those that did

523 not. We also selected two collection points to optimize the ratio of mRNA at two collection

524 points for each experimental group. The final experimental protocol is shown in Figure $1 \mathrm{C}$.

525 Adult female Metazygia wittfeldae were collected on 16 July 2018 in Washington County, TN

526 and placed in clear plastic deli jars on a lab bench receiving natural day, night lighting. All

527 spiders were provided with a wet cotton ball and offered a small cricket. On 18 July 2018, each

528 spider was moved to a clear glass or plastic tube (25 mm diameter x $15 \mathrm{~cm}$ length) and inserted 
529 into a locomotor activity monitor (model LAM25, Trikinetics Inc., Waltham, Massachusetts,

530 U.S.A.). The monitors were placed into two separate temperature-controlled $\left(24 \pm 0.5^{\circ} \mathrm{C}\right)$

531 environmental chambers with the lights on (1400-1600 lux) at 16:00 until 20:00, after which

532 the spiders remained in the dark for 3.5 days ( 84 hours) to establish the free-running period of

533 each individual. In order to synchronize the circadian rhythms of the individual spiders, they

534 were then entrained to a $12: 12 \mathrm{LD}$ cycle for 5 days. On the evening of the $5^{\text {th }}$ day the lights went

535 out at 20:00 (et0), as per the prior 4 days. However, one chamber received 1 hour of light (1400-

5361600 lux) starting 5 hours after the lights were turned off (et5-et6, 01:00-02:00 27 July 2018)

537 (pulse group). The other chamber remained dark (no pulse group). One hour after the end of the

538 light pulse (et7, 03:00) 5 spiders were removed from each environmental chamber and snap

539 frozen in liquid nitrogen under dim red light. Both chambers remained dark until the final

540 collection time 9 hours later (et16, 12:00), when 5 spiders were again removed from each

541 chamber and snap frozen in liquid nitrogen under infrared light.

542

543 RNA-sequencing, transcriptome assembly, and annotation

544 Total RNA was isolated from one half of a cephalothorax (the fused head-body) of each of the

54520 experimental spiders by homogenizing tissue in TRIzol (Invitrogen) and further purifying

546 with the RNeasy Mini Kit (Qiagen) with on-column DNaseI digestion to remove contaminating

547 DNA. RNA quality and quantity were verified with the Agilent 2100 Bioanalyzer and 20

548 individually barcoded cDNA libraries were constructed with the TruSeq kit (Illumina) by the

549 Genomics Research Laboratory at the Biocomplexity Institute, Virginia Tech. All 20 libraries

550 were multiplexed and sequenced in a single lane with the NextSeq 500 (Illumina) using the High 
551 Output mode with 300 cycles (150 base paired end reads). De-multiplexing and barcode removal 552 were performed by Virginia Tech.

553 Transcripts were de novo assembled with Trinity v2.8.4 [43] from all 20 RNAseq libraries.

554 Raw reads were trimmed of low quality base calls using Trimmomatic [44] and in silico

555 normalization was used to reduce the number of reads entering the assembly phase, as per scripts 556 in Trinity v2.8.4.

557 Annotation involved a multi-step process using the Trancriptome Trimming and Annotation 558 Pipeline (TrTAP, [45]). In brief, initial transcript abundance was estimated using RSEM v.1.3.1

559 [20]. Trimming and annotation then started with any transcripts with a significant BLASTN 560 match to the SILVA ribosomal database v132 [46] or a tRNAScan v.1.3.1 match [47] were

561 identified. Second, all the transcripts were compared to a custom-made database of spider silk 562 proteins encoded by the spidroin gene family [48], and subsequently to the proteomes of 5 563 arthropod species using BLASTx. These species included 3 spiders (listed from most closely 564 related to least): Araneus ventricosus (GCA_013235015.1_Ave_3.0, Kono et al. 2019)),

565 Trichonephila clavipes (MWRG00000000.1, [50]), and Stegodyphus mimosarum

566 (GCA_000611955, [51]); another arachnid, Ixodes scapularis (GCF_016920785.1,Gulia-Nuss et 567 al. 2016); and an insect, Drosophila melanogaster (FB2019_08 [53]) The Drosophila gene 568 matches with a cutoff of 1e-40 were used to identify chimeric sequences with a python script 569 described in [54].

$570 \quad$ For each Trinity "gene", the transcript with the best BLAST alignment, alignment as 571 determined by the highest bit-score, or with the longest open reading frame (ORF) if there were 572 no BLAST alignments, was chosen to represent that gene. To further reduce redundancy and 573 identify fragments of genes, the best matching gene of each protein in the curated silk gene 
574 database plus the 5 species proteomes were also identified. These reciprocal best matches were

575 always retained for downstream analyses (BEST, Table 1). If a Trinity gene was not the best

576 match of a database protein it was considered "GOOD" if it aligned to $>20 \%$ of the database

577 protein and exceeded 1 TPM (transcript per million) in at least one RNAseq library. Genes that

578 aligned to $>20 \%$ of the database protein and did not meet the 1 TPM threshold were dubbed

579 "LOW_EXP" (Table 1). Genes that aligned to $<20 \%$ of the database proteins were dubbed

580 "LOW_COV" (Table 1). For the genes with no BLAST alignments to a proteome, the gene was

581 called "LONGORF" if the ORF exceeded 50 amino acids and had $>1$ TPM in at least one

582 RNAseq library (Table 1). ORFs that exceeded 50 amino acids but had <1 TPM were called

583 “LOW_EXP_LONGORF” (Table 1). A reduced set of probable protein-coding genes (BEST,

584 GOOD, LONGORF, and LOWEXP) was then translated using the direction and frame of the

585 matching BLASTX hit, or in cases without such hit, translated according to the longest open

586 reading frame. These translated genes were further annotated by comparison to SwissProt with

587 BLASTP, and to PFAM with HMMER v 3.2.1[55] with Gene Ontology (GO) terms [56, 57]

588 assigned to each "gene" based on the best alignment to Drosophila melanogaster, SwissProt, and

589 PFAM, with GO SLIM annotations obtained from GO SLIM viewer [58]. The completeness of

590 the reduced transcriptome was assessed with the set of single copy orthologous genes in

591 arthropods (BUSCO v3, [19]).

592

593 Canonical circadian gene identification

594 Our initial BLAST results identified multiple transcripts with significant alignments to the

595 canonical circadian genes: $C l k, c y c$, tim, per, dbt, cryl, cry2. Since these genes are each part of

596 larger multi-paralog gene families, we used a phylogenetic approach to determine which 
transcript was an ortholog of the canonical clock gene in D. melanogaster or Apis mellifera. We

598 first used Orthofinder v2. [59] to identify groups of homologous genes among 6 spider species, a

599 tick, and two insects. In addition to our M. wittfeldae transcriptome, we used the predicted

600 proteomes from published transcriptomes or genomes for the following species: Araneus

601 ventricosus (Araneidae, BGPR01000000.1), Trichonephila clavipes (Araneidae,

602 MWRG01000000.1), Latrodectus hesperus (Theridiidae, GBJN01000000.1), Parasteatoda

603 tepidariorum (Theridiidae, GCF_000365465.3), Stegodyphus mimosarum (Eresidae,

604 GCA_000611955.2), Ixodes scapularis (Ixodidae, GCF_016920785.1), D. melanogaster

605 (Drosophilidae, FB2019_08), A. mellifera (Apidae, GCF_003254395.1). We then identified the

606 "orthogroups" containing the canonical circadian gene of D. melanogaster or A. mellifera (Table

607 4). For 4 of the genes, only a single $M$. wittfeldae transcript was placed in the orthogroup; these

608 were considered the ortholog of the circadian gene. For Doubletime, $d b t$, and the cryptochromes

609 (cryl and cry2), we inferred phylogenetic trees for their orthogroups using maximum likelihood

610 (RAxML,[60]. From the resulting trees, we chose the single $M$. wittfeldae transcripts in the clade

611 with the canonical circadian clock gene (Additional File 2: Figures S4-S5).

612

\begin{tabular}{|l|l|l|l|}
\hline \multicolumn{2}{|l|}{ Table 4. Circadian “clock" gene identification. } \\
\hline ID & Drosophila Gene(s) & Orthogroup ID & Metazygia Gene \\
\hline Cycle & FBpp0074693 & OG0006887 & MW_TRINITY_DN59140_c0_g1 \\
\hline \multirow{2}{*}{ Clock } & FBpp0076500 FBpp0099478 FBpp0099480 & & \\
\hline & FBpp0306709 FBpp0306710 & OG0003118 & MW_TRINITY_DN2943_c0_g1 \\
\hline Doubletime & FBpp0306615 FBpp0422974 & OG0001802 & MW_TRINITY_DN4945_c0_g1 \\
\hline Period & FBpp0070455 FBpp0304590 & OG0004797 & MW_TRINITY_DN146_c0_g1 \\
\hline
\end{tabular}




\begin{tabular}{|l|l|l|l|}
\hline Timeless & FBpp0082180 & OG0012214 & MW_TRINITY_DN42615_c0_g1 \\
\hline \multirow{2}{*}{ CRY1 } & FBpp0080934 FBpp0080935 FBpp0083150 & & MW_TRINITY_DN18046_c0_g1 \\
& FBpp0307793 FBpp0307794 & OG0001358 & MW_TRINITY_DN308_c1_g1 \\
\hline CRY2 & Apis mellifera protein: & OG0001358 & MW_TRINITY_DN308_c1_g1 \\
\hline The circadian genes (bold) were identified for D. melanogaster from FlyBase v2019_08. In cases where more \\
than one M. wittfeldae transcript was in an orthogroup with a circadian gene, phylogenetic trees were examined \\
for orthology (Additional File 2: Figures S4-S5). The ortholog is bolded. \\
\hline
\end{tabular}

\section{Differential gene expression analysis}

615 Differential gene expression among experimental groups was based on gene-level expression

616 estimates derived from transcript abundance estimates as recommended by [61]. In brief, the

617 expected read counts of each Trinity-assembled transcript were calculated by RSEM v.1.3.1 [20],

618 which takes into account read-mapping ambiguity due to multiple isoforms or even alleles

619 having been assembled. The gene-level counts were then calculated as the sum of the included

620 transcripts, weighted by their length as described in Soneson et al. [61], and implemented

621 through Trinity v2.8.4. Because RSEM can distribute one read among multiple transcripts, some

622 counts were not integer values. These values were rounded down for input into differential

623 expression analyses, which require integer values for read counts. Prior to further expression

624 analyses, genes unlikely to encode proteins were removed (RNA, CHIMERA, LOW_COV, and

625 NO_HITS, Table 1). For comparing expression levels among circadian genes, we used the TPM

626 normalized for differences in sequencing depth among libraries using the Trimmed Mean of M

627 (TMM) values calculated with Trinity. 
Four different pairwise gene expression analyses were performed on the read count data

629 using the 'EdgeR' Bioconductor package [62]: 1) comparing samples with light pulse to samples

630 without light pulse at et7; 2) comparing samples with light pulse to samples without light pulse at

631 et16; 3) comparing samples collected at et7 to samples collected at et16 with a light pulse; 4)

632 comparing samples collected at et7 to samples collected at et16 without a light pulse. A gene is

633 kept in the analysis if it is sufficiently expressed $(\mathrm{CPM}>0.1)$ in at least two samples. The raw

634 library sizes were normalized using the TMM method from EdgeR. The significantly

635 differentially expressed genes in the pair-wise comparisons were identified using false discovery

636 rate $(\mathrm{FDR})<0.05$ and absolute value of $\log \mathrm{FC}>1$ (Additional file 1: Table $\mathrm{S} 1$ ).

\section{Gene Ontology analysis}

639 GO analysis was conducted to determine the functions enriched in DE genes relative to all

640 annotated genes using the 'GOseq' Bioconductor package [63] based on Wallenius non-central

641 hyper-geometric distribution, with an over represented p-value of 0.1 . The lists of GO terms

642 (Additional file 4: Table S3) along with their p-values generated from 'Goseq' were summarized

643 and visualized by REViGO online tool [64]. The semantically similar terms of the identified GO

644 terms were removed using SimRel semantic clustering. Scatterplots of the remaining terms after

645 the redundancy reduction were generated using the source code from REViGO, which plotted the 646 terms in a two-dimensional space by applying multidimensional scaling to a matrix of the GO

647 terms' semantic similarities (Figure 4) [64].

648

649 Data visualization and graphical output generation 
650 In order to check the dissimilarities among the samples, MDS plots (Additional File 2, Figure

651 S1) were generated using the 'limma' Bioconductor R package [65]. The distance between each

652 pair of samples is calculated as the leading fold change, defined as the root-mean-square of the

653 largest $500 \log 2$-fold changes between that pair of samples.

654 Venn diagrams (Figure 3) were generated to identify shared and unique DE genes between

655 various contrast groups based on the DE analysis results (Additional File 1: Table S1) using the 656 freely available VENNY 2.1 online tool [66].

657 Bar plots of up- and down- regulated DEGs, volcano plots of DEGs as well as scatterplots of $658 \operatorname{logFC}$ values (Additional File 2: Figure S2; Figure 5) were generated using the 'ggplot2' R 659 package [67].

660

661 List of abbreviations

662 BP: Biological Process

663 CC: Cellular Component

664 Clk: Clock

665 CPM: Counts Per Million

666 Cry1: Cryptochrome 1

667 Cry2: Cryptochrome 2

668 Cyc: Cycle

669 Dbt: Doubletime

670 DD: Constant darkness

671 DE: Differentially expressed

672 Et7: The group collected 7 hours after the last light phase 
673 Et16: The group collected 16 hours after the last light phase

674 FC: Fold Change

675 FDR: False Discovery Rate

676 FRP: Free-running period

677 GO: Gene Ontology

678 LD: Light-dark environments

679 MDS: Multidimensional scaling

680 MF: Molecular Function

681 No pulse: The group receiving no light pulse

682 ORF: Open reading frame

683 PDEA: pairwise differential expression analysis

684 Per: Period

685 Pulse: The group receiving a light pulse

686 Tim: Timeless

687 TPM: Transcripts Per Million

688 TMM: Trimmed mean of M values

689

690 Declarations

691 - Ethics approval and consent to participate

692 The experimental animals in this study, Metazygia wittfeldae, are not regulated.

693 - Consent for publication 
694 Not applicable.

695 - Availability of data and materials

696 The datasets supporting the conclusions of this article are available within the article (and its

697 additional files) and in NCBI under the Short Read Archive (SRR16156593- SRR16156612) and

698 Transcriptome Shotgun Assembly (TSA: GJLH00000000).

699 - Competing interests

700 The authors declare that they have no competing interests.

$701 ・$ Funding

702 This work was supported by Washington and Lee University through Summer Lenfest Research

703 Grants to NT and NAA and Summer Research Scholarships to AM and CSAL, the National

704 Science Foundation [Award number IOS-1755142 to NAA], and Eastern Tennessee State

705 University through a Research and Development Committee Major Grant.

706 - Authors' contributions

707 NT, AM, TCJ, DM, and NAA conceived and designed the experiments. AM, TCJ, DM, and

708 NAA collected the data. NT and AM and developed the mathematical model. NT, WC, LQ, TC,

709 and NAA analyzed the data. All authors read and approved the final manuscript.

710 - Acknowledgements 
711 We thank Hyun Song and C.S. Adrian Lam for assistance with early analyses. We also thank

712 Madeleine Miller for help collecting spiders.

713 - Authors' information (optional)

714

\section{Bibliography}

716 1. Edery I. Circadian rhythms in a nutshell. Physiol Genomics. 2000;3:59-74.

717 2. Wager-Smith K, Kay SA. Circadian rhythm genetics: from flies to mice to humans. Nat Genet. 718 2000;26:23-7.

719 3. DeCoursey PJ. The behavioral ecology and evolution of biological timing systems. In:

720 Chronobiology: Biological timekeeping. Sunderland, MA, US: Sinauer Associates; 2004. p. 27 72165.

722 4. Johnson $\mathrm{CH}$. Precise circadian clocks in prokaryotic cyanobacteria. Curr Issues Mol Biol.

$7232004 ; 6: 103-10$.

724 5. Refinetti R. Comparison of light, food, and temperature as environmental synchronizers of the 725 circadian rhythm of activity in mice. J Physiol Sci. 2015;65:359-66.

726 6. Golombek DA, Rosenstein RE. Physiology of circadian entrainment. Physiol Rev.

727 2010;90:1063-102.

728 7. Zeng H, Qian Z, Myers MP, Rosbash M. A light-entrainment mechanism for the Drosophila

729 circadian clock. Nature. 1996;380:129-35.

730 8. Yuan Q, Metterville D, Briscoe AD, Reppert SM. Insect cryptochromes: gene duplication and 731 loss define diverse ways to construct insect circadian clocks. Mol Biol Evol. 2007;24:948-55.

732 9. Zhu H, Sauman I, Yuan Q, Casselman A, Emery-Le M, Emery P, et al. Cryptochromes define 733 a novel circadian clock mechanism in monarch butterflies that may underlie sun compass

734 navigation. PLoS Biol. 2008;6. doi:10.1371/journal.pbio.0060004.

735 10. Moore D, Watts JC, Herrig A, Jones TC. Exceptionally short-period circadian clock in 736 Cyclosa turbinata: regulation of locomotor and web-building behavior in an orb-weaving spider. 737 J Arachnol. 2016;44:388-96.

738 11. Ralph MR, Menaker M. A mutation of the circadian system in golden hamsters. Science.

$7391988 ; 241: 1225-7$. 
12. Konopka RJ, Benzer S. Clock mutants of Drosophila melanogaster. Proc Natl Acad Sci. 1971;68:2112-6.

742 13. Mah A, Ayoub N, Toporikova N, Jones TC, Moore D. Locomotor activity patterns in three 743 spider species suggest relaxed selection on endogenous circadian period and novel features of

744 chronotype. J Comp Physiol A. 2020;206:499-515.

745 14. Jones TC, Wilson RJ, Moore D. Circadian rhythms of locomotor activity in Metazygia wittfeldae (Araneae: Araneidae). J Arachnol. 2018;46:26-30.

15. Czeisler CA, Duffy JF, Shanahan TL, Brown EN, Mitchell JF, Rimmer DW, et al. Stability, precision, and near-24-Hour period of the human circadian pacemaker. Science. 1999;284:217781.

16. Ebihara S, Tsuji K, Kondo K. Strain differences of the mouse's free-running circadian rhythm in continuous darkness. Physiol Behav. 1978;20:795-9.

753 B Biol Sci. 2010;277:3335-42.

754 18. Shimizu T, Masaki S. Geographical and species variation in circadian rhythm parameters in 755 nemobiine crickets. Physiol Entomol. 1997;22:83-93.

19. Waterhouse RM, Seppey M, Simão FA, Manni M, Ioannidis P, Klioutchnikov G, et al. BUSCO Applications from quality assessments to gene prediction and phylogenomics. Mol Biol 758 Evol. 2018;35:543-8.

759 20. Li B, Dewey CN. RSEM: accurate transcript quantification from RNA-Seq data with or 760 without a reference genome. BMC Bioinformatics. 2011;12:323.

761 21. Robinson MD, McCarthy DJ, Smyth GK. edgeR: a Bioconductor package for differential 762 expression analysis of digital gene expression data. Bioinformatics. 2010;26:139-40.

763 22. Huntley RP, Sawford T, Mutowo-Meullenet P, Shypitsyna A, Bonilla C, Martin MJ, et al. 764 The GOA database: Gene Ontology annotation updates for 2015. Nucleic Acids Res.

765 2015;43:D1057-63.

766

767

768

769

770

771

772

773
23. Ceriani MF, Hogenesch JB, Yanovsky M, Panda S, Straume M, Kay SA. Genome-wide expression analysis in Drosophila reveals genes controlling circadian behavior. J Neurosci. 2002;22:9305-19.

24. Ueda HR, Matsumoto A, Kawamura M, Iino M, Tanimura T, Hashimoto S. Genome-wide transcriptional orchestration of circadian rhythms in Drosophila. J Biol Chem. 2002;277:1404852.

25. Claridge-Chang A, Wijnen H, Naef F, Boothroyd C, Rajewsky N, Young MW. Circadian regulation of gene expression systems in the Drosophila head. 2001;32:657-71. 
26. Keegan KP, Pradhan S, Wang J-P, Allada R. Meta-analysis of Drosophila circadian microarray studies identifies a novel set of rhythmically expressed genes. PLOS Comput Biol. 2007;3:e208.

27. Abraham U, Granada AE, Westermark PO, Heine M, Kramer A, Herzel H. Coupling governs entrainment range of circadian clocks. Mol Syst Biol. 2010;6:438.

28. Brown SA, Kunz D, Dumas A, Westermark PO, Vanselow K, Tilmann-Wahnschaffe A, et al. Molecular insights into human daily behavior. Proc Natl Acad Sci U S A. 2008;105:1602-7.

29. Kurosawa G, Goldbeter A. Amplitude of circadian oscillations entrained by 24-h light-dark cycles. J Theor Biol. 2006;242:478-88.

30. Vaze KM, Helfrich-Förster C. Drosophila ezoana uses an hour-glass or highly damped circadian clock for measuring night length and inducing diapause. Physiol Entomol. 2016;41:378-89.

31. Gentile C, Rivas GBS, Meireles-Filho ACA, Lima JBP, Peixoto AA. Circadian expression of clock genes in two mosquito disease vectors: cry2 Is different. J Biol Rhythms. 2009;24:444-51.

32. Weger BD, Sahinbas M, Otto GW, Mracek P, Armant O, Dolle D, et al. The light responsive transcriptome of the zebrafish: function and regulation. PLoS ONE. 2011;6:e17080.

33. Rugnone ML, Faigon Soverna A, Sanchez SE, Schlaen RG, Hernando CE, Seymour DK, et al. LNK genes integrate light and clock signaling networks at the core of the Arabidopsis oscillator. Proc Natl Acad Sci. 2013;110:12120-5.

34. Chen Y-R, Wei W-L, Tzeng DTW, Owens ACS, Tang H-C, Wu C-S, et al. Effects of artificial light at night (ALAN) on gene expression of Aquatica ficta firefly larvae. Environ Pollut. 2021;281:116944.

35. Lee J-E, Edery I. Circadian regulation in the ability of Drosophila to combat pathogenic infections. Curr Biol CB. 2008;18:195-9.

36. McDonald MJ, Rosbash M. Microarray analysis and organization of circadian gene expression in Drosophila. Cell. 2001;107:567-78.

37. Bedrosian TA, Fonken LK, Walton JC, Nelson RJ. Chronic exposure to dim light at night suppresses immune responses in Siberian hamsters. Biol Lett. 2011;7:468-71.

38. Durrant J, Michaelides EB, Rupasinghe T, Tull D, Green MP, Jones TM. Constant illumination reduces circulating melatonin and impairs immune function in the cricket Teleogryllus commodus. PeerJ. 2015;3:e1075.

39. McLay LK, Nagarajan-Radha V, Green MP, Jones TM. Dim artificial light at night affects mating, reproductive output, and reactive oxygen species in Drosophila melanogaster. J Exp Zool Part Ecol Integr Physiol. 2018;329:419-28. 
40. Willmott NJ, Henneken J, Selleck CJ, Jones TM. Artificial light at night alters life history in a nocturnal orb-web spider. PeerJ Inc.; 2018. doi:10.7287/peerj.preprints.26943v1.

42. Leloup J-C, Gonze D, Goldbeter A. Limit cycle models for circadian rhythms based on transcriptional regulation in Drosophila and Neurospora. J Biol Rhythms. 1999;14:433-48.

813 43. Grabherr MG, Haas BJ, Yassour M, Levin JZ, Thompson DA, Amit I, et al. Trinity: reconstructing a full-length transcriptome without a genome from RNA-Seq data. Nat

815 Biotechnol. 2011;29:644-52.

816 44. Bolger AM, Lohse M, Usadel B. Trimmomatic: a flexible trimmer for Illumina sequence 817 data. Bioinformatics. 2014;30:2114-20.

818 45. Clarke TH. TrTAP: Transcriptome Trimming and Annotation Pipeline. https://github.com/thclarke/TrTAP.

820 46. Quast C, Pruesse E, Yilmaz P, Gerken J, Schweer T, Yarza P, et al. The SILVA ribosomal RNA gene database project: improved data processing and web-based tools. Nucleic Acids Res. 2013;41 Database issue:D590-6.

47. Chan PP, Lowe TM. tRNAscan-SE: Searching for tRNA genes in genomic sequences.

824 Methods Mol Biol Clifton NJ. 2019;1962:1-14.

825 48. Ayoub NA, Friend K, Clarke T, Baker R, Correa-Garhwal S, Crean A, et al. Protein 826 composition and associated material properties of cobweb spiders' gumfoot glue droplets. Integr 827 Comp Biol. 2021. doi:10.1093/icb/icab086.

49. Kono N, Nakamura H, Ohtoshi R, Moran DAP, Shinohara A, Yoshida Y, et al. Orb-weaving spider Araneus ventricosus genome elucidates the spidroin gene catalogue. Sci Rep. 2019;9:8380.

50. Babb PL, Lahens NF, Correa-Garhwal SM, Nicholson DN, Kim EJ, Hogenesch JB, et al. The Nephila clavipes genome highlights the diversity of spider silk genes and their complex expression. Nat Genet. 2017;49:895-903. genomes provide insight into composition and evolution of venom and silk. Nat Commun.

837 52. Gulia-Nuss M, Nuss AB, Meyer JM, Sonenshine DE, Roe RM, Waterhouse RM, et al. 838 Genomic insights into the Ixodes scapularis tick vector of Lyme disease. Nat Commun. 2016;7. 839 doi:10.1038/ncomms10507. 
54. Clarke TH, Garb JE, Hayashi CY, Haney RA, Lancaster AK, Corbett S, et al. Multi-tissue transcriptomics of the black widow spider reveals expansions, co-options, and functional processes of the silk gland gene toolkit. BMC Genomics. 2014;15:365.

55. Eddy S. HMMER: biosequence analysis using profile hidden Markov models. www.hmmer.org.

56. Ashburner M, Ball CA, Blake JA, Botstein D, Butler H, Cherry JM, et al. Gene Ontology: tool for the unification of biology. Nat Genet. 2000;25:25-9.

57. Gene Ontology Consortium. The Gene Ontology resource: enriching a GOld mine. Nucleic Acids Res. 2021;49:D325-34.

58. McCarthy FM, Wang N, Magee GB, Nanduri B, Lawrence ML, Camon EB, et al. AgBase: a functional genomics resource for agriculture. BMC Genomics. 2006;7:229.

59. Emms DM, Kelly S. OrthoFinder: phylogenetic orthology inference for comparative genomics. Genome Biol. 2019;20:238.

60. Stamatakis A. RAxML version 8: a tool for phylogenetic analysis and post-analysis of large phylogenies. Bioinformatics. 2014;30:1312-3.

61. Soneson C, Love MI, Robinson MD. Differential analyses for RNA-seq: transcript-level estimates improve gene-level inferences. F1000Research. 2016;4:1521.

62. Robinson MD, Oshlack A. A scaling normalization method for differential expression analysis of RNA-seq data. Genome Biol. 2010;11:R25.

63. Young MD, Wakefield MJ, Smyth GK, Oshlack A. Gene ontology analysis for RNA-seq: accounting for selection bias. Genome Biol. 2010;11:R14.

64. Supek F, Bošnjak M, Škunca N, Šmuc T. REVIGO summarizes and visualizes long lists of gene ontology terms. PLOS ONE. 2011;6:e21800.

65. Ritchie ME, Phipson B, Wu D, Hu Y, Law CW, Shi W, et al. limma powers differential expression analyses for RNA-sequencing and microarray studies. Nucleic Acids Res. 2015;43:e47-e47.

66. Oliveros J. Venny 2.1.0. https://bioinfogp.cnb.csic.es/tools/venny/. Accessed 9 Oct 2021.

67. Hadley Wickham. ggplot2: Elegant Graphics for Data Analysis. Springer-Verlag New York; 2016. https://ggplot2.tidyverse.org. 


\section{Supplementary Files}

This is a list of supplementary files associated with this preprint. Click to download.

- AdditionalFile1TableS1.xlsx

- Additionalfile2SuppFigures.docx

- AdditionalFile3Tables2.csv

- AdditionalFile4TableS3.xlsx

- AdditionalFile5TableS4.xlsx 